\title{
ANTIDEPRESSANTS AS AN ADD-ON STRATEGY FOR NEGATIVE SYMPTOMS A REVIEW AND A CASE-SERIES USING VORTIOXETINE
}

\author{
19th WPC WORLD CONGRESS OF PSYCHIATRY. Lisbon, Portugal 21-24 August, 2019 \\ D. Esteves-Sousa 1; J. Gago 2; S. Gomes-Pereira 2; M. Albuquerque 1; J. Facucho-Oliveira 1; P. Espada ${ }^{1}$, L. Mendonça ${ }^{1}$ \\ 1 - Hospital de Cascais, Departamento de Psiquiatria e Saúde Mental. \\ 2 - Centro Hospitalar Lisboa Ocidental, Departamento de Psiquiatria e Saúde Mental.
}

\section{BACKGROUND AND OBJECTIVES}

Schizophrenia (SCZ) is a heterogeneous clinical syndrome comprising a number of psychopathology domains, that vary from patient to patient. It affects around $0.5-0.8 \%$ of people worldwide. It is characterized by positive and negative symptoms (NSS) and often by cognitive impairment ${ }^{[1]}$.

NSS are characterized by diminished emotional expression, avolition, alogia, anhedonia, social withdrawal and poor social drive $[2,3]$. The overwhelming majority of patients suffers to some extent from this problem, which persists for most of their lives and promote marked functional disability [1]. An adequate treatment would mean an important progress. Indeed, multiple clinical and pharmacological interventions have been the subject of investigation, but to this point, the efficacy of actual treatments is questionable ${ }^{[4]}$.

Antidepressants (AD), including selective serotonin reuptake inhibitors (SSRI), are one of the promising approaches. This class seems to have the additional benefits of being safe and relatively innocuous ${ }^{[5]}$.

Vortioxetine, a new SSRI, has a unique receptor binding profile and pharmacodynamic properties that provided a non-clinical rationale to investigate its use on NSS (see Fig 1).



* Human isoforms

Fig. 1 - Vortioxetine binding profile and pharmacodynamic properties 6,7.

Mechanism of action and its multimodal approach $[7,8]$ :

a) SERT receptor antagonism actions

b) $5 \mathrm{H} T 1 \mathrm{~A}$ receptor agonism actions

Increase oxytocin release; Increase dopamine release in the medial prefrontal cortex, striatum, and hippocampus; Ameliorate SSRI's adverse effects (sexual disfunction, emotional blunting, ...).

c) 5 HT1B receptor partial agonism actions

Desensitizing 5-HT1B receptors - increase in 5-HT levels; modulation of other neurotransmitters across the brain.

d) 5 HT3 receptor antagonism actions

Studies show efficacy for NSS - modulation of mesolimbic and mesocortical dopaminergic activity.

e) 5 HT7 receptor antagonism actions

Regulation of circadian rhythm, sleep, cognition and mood.

Objectives:

- Our aim is to review the literature published to date about the add-on strategy with antidepressants for the treatment of negative symptoms (NSS) and present a case-series with adjunctive use of vortioxetine.

\section{MATERIALS AND METHODS}

Non-systematic review of the literature in scientific databases Google Scholar and Pubmed. The case-series of seven patients with stable schizophrenia treated with vortioxetine were described with data retrieved from the clinical files. NSS were assessed using Clinical Assessment Interview For Negative Symptoms (CAINS).

\section{RESULTS}

\section{Antidepressants in negative symptoms of Schizophrenia:}

The literature shows that antidepressants appear to be safe and relatively innocuous in SCZ, with a low risk of exacerbation of psychosis [5]. A large metaanalysis of the efficacy for NSS was recently published, including 48 trials and 1905 participants. A highly significant but modest effect size improvement was seen (SMD -0.3) overall $[4,5]$. Larger effects were seen in a subgroup described as having predominantly negative symptoms at baseline (SMD -0.58) ${ }^{[4,5]}$. Most guidelines, at some point, recommend the use of $\mathrm{AD}$ as an add-on strategy ${ }^{[4,5]}$.

\section{Case-series using Vortioxetine as an add-on strategy for NSS:}

The sample group was characterized by seven outpatients with SCZ diagnosed in less than five years, compensated and stable, without therapeutic for at least 3 months. Patients were screened with Hamilton Depression Rating Scale (HAM-D) and Simpson-Angus Extrapyramidal side effects scale (SAS) to exclude significant depressive or extrapyramidal symptoms. Patients were started with vortioxetine $10 \mathrm{mg} /$ day for eight weeks and then $20 \mathrm{mg} /$ day for the next four weeks, unless side effects appeared.

Clinical assessment of the group showed a significant reduction in negative symptoms in the mean CAINS score from $37,9 \pm 4,5$ to $24,3 \pm 5$ in twelve weeks $(P<0,01)$ - see Graph 1 and 2. The reduction was also observed clinically. No major side effects or psychosis exacerbation was observed.

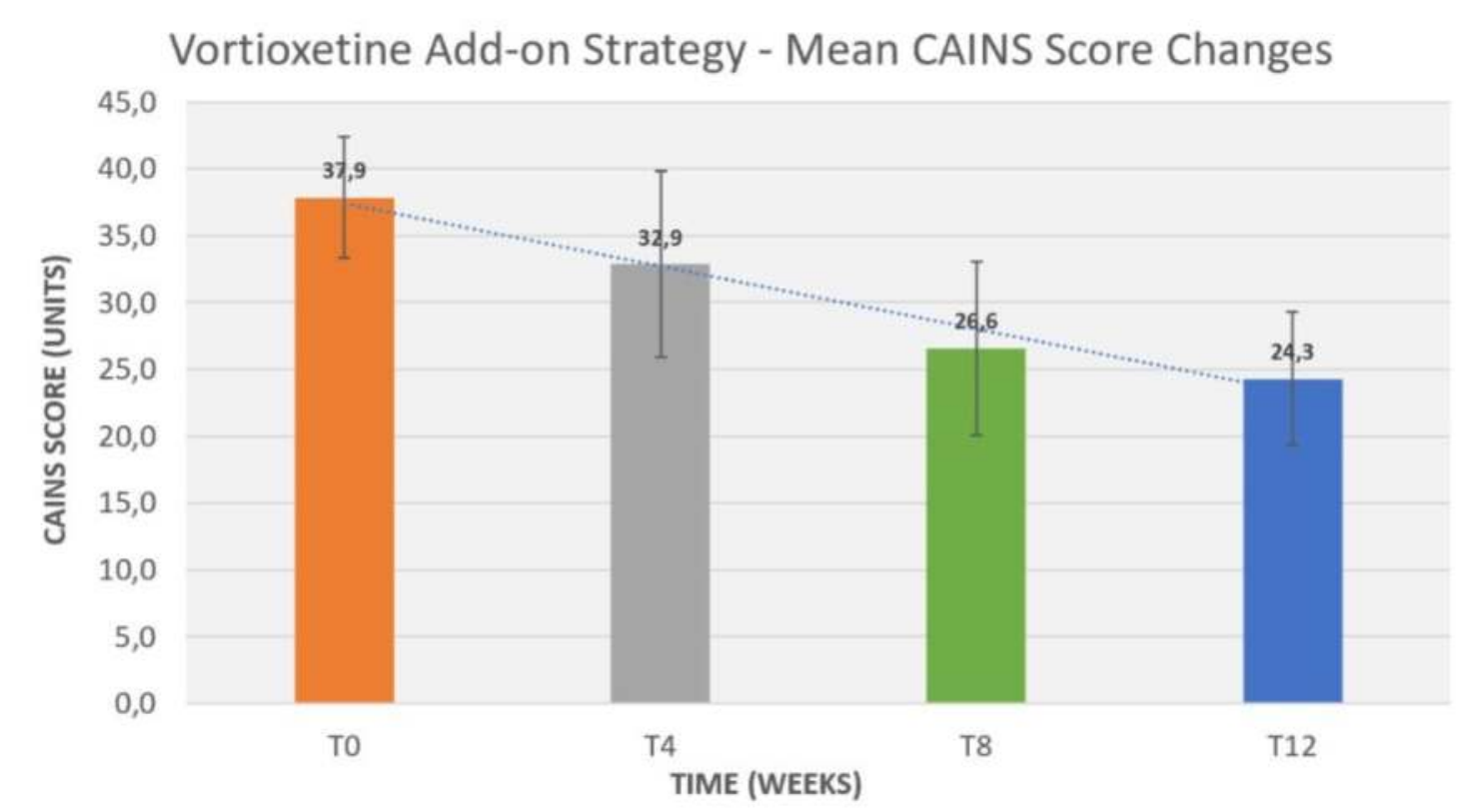

Graph 1 - Case-series of 7 patients with stable SCZ with less than five years of disease and without therapeutic changes for at least 3 months. Patients started with vortioxetine $10 \mathrm{mg} /$ day for eight weeks and then $20 \mathrm{mg} /$ day for the next four weeks.

Vortioxetine Add-on Strategy - CAINS Score per Patient

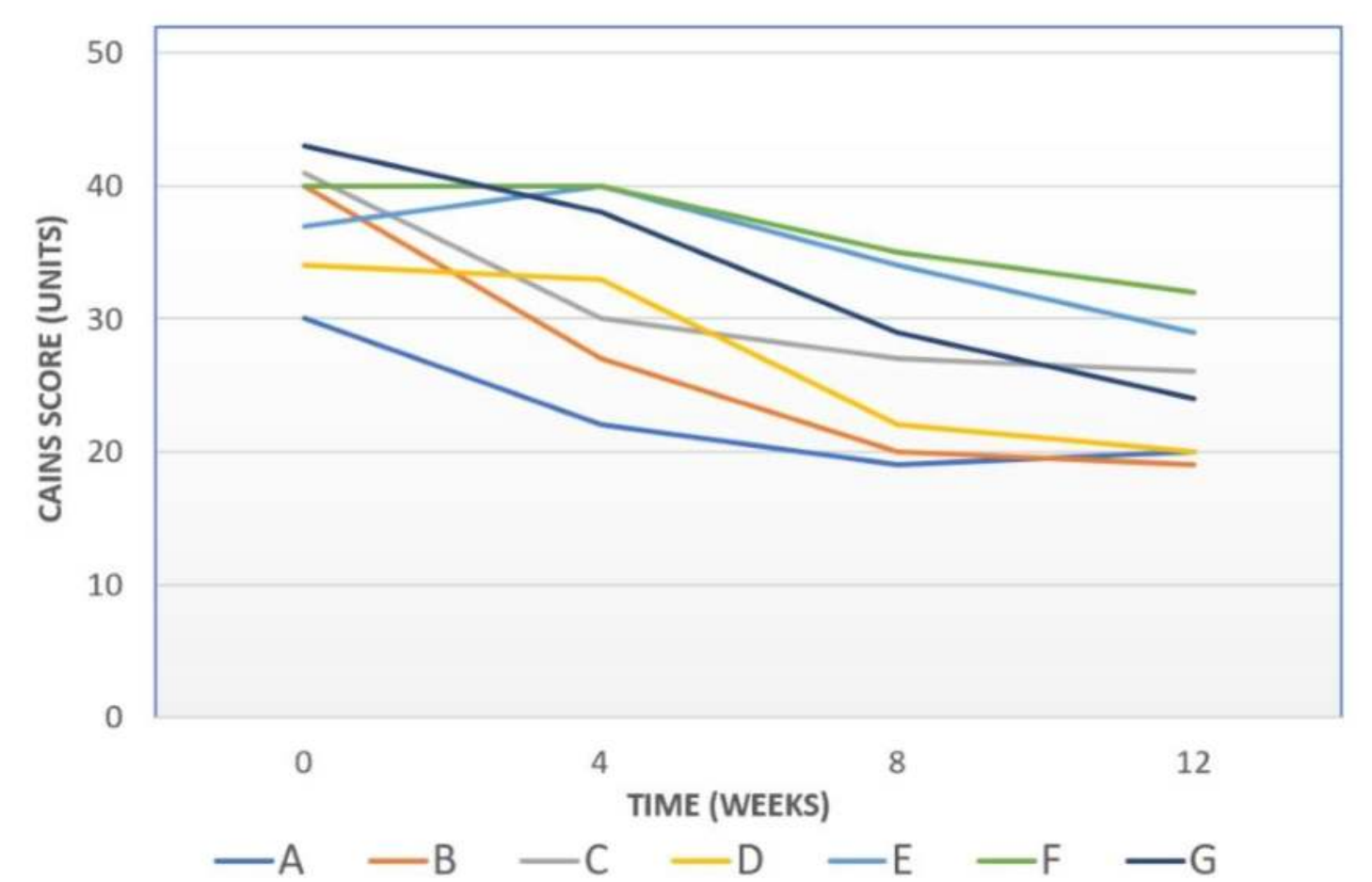

Graph 2 - Changes of CAINS Score per patient with stable SCZ over twelve weeks of vortioxetine.

\section{CONCLUSIONS}

There is some data supporting modest benefits in the use of antidepressants in NSS and no relevant data specifically for vortioxetine. The significative change in NSS of the group shows a promising effect. Further clinical studies will help clarify this potential therapeutic role. 